\title{
Characteristics and Outcomes of Adults Hospitalized with SARS-COV-2 Community-Acquired Pneumonia in Louisville, Kentucky
}

\author{
Julio Ramirez ${ }^{1,2 *}$; Jose Bordon²; Rodrigo Cavallazzi' ; Stephen Furmanek ${ }^{2}$; Amr Aboelnasr ${ }^{2}$; Mahder Tella ${ }^{2}$; William Mattingly ${ }^{2}$; Ashley Wilde ; $^{3}$ \\ Daisy Sangroula ${ }^{1}$; Demetra Antimisiaris ${ }^{1}$; Donghoon Chung ${ }^{1}$; Guillermo Cabral; Rafael Fernandez-Botran ${ }^{1}$; Jiapeng Huang ${ }^{1}$; Martin Gnoni ${ }^{2}$; \\ Ozan Akca, ${ }^{4}$; Paul Schulz ${ }^{6}$; Phillip Bressoud ${ }^{1}$; Priya Krishnan ${ }^{7}$; Sally Suliman ${ }^{8}$; Sathya S. Krishnasamy ${ }^{8}$; Stephen Hanson ${ }^{2}$; William P. \\ McKinney $^{1}$; Bryan Moffett ${ }^{2}$; Leslie Wolf ${ }^{2}$ : Mark Burns ${ }^{2}$; Ruth Carrico ${ }^{2}$; Forest Arnold²; and the CERID COVID-19 Study Group \\ ${ }^{1}$ University of Louisville, Louisville, KY, USA; ${ }^{2}$ Division of Infectious Diseases, University of Louisville, KY, USA; ${ }^{3}$ Norton Healthcare, Louisville, KY, USA; ${ }^{4}$ Vice Chair, \\ Professor, Department of Anesthesiology \& Perioperative Medicine, Louisville, KY, USA; ${ }^{5}$ Director, Stroke ICU, CCSCRP, University of Louisville, Louisville, KY, USA; \\ ${ }^{6}$ Infectious Diseases, Norton Healthcare, Louisville, KY, USA; ${ }^{7}$ Robley Rex VA Medical Center, Louisville, KY, USA; ${ }^{8}$ School of Medicine, University of Louisville, \\ Louisville, KY, USA \\ *j.ramirez@Louisville.edu
}

Recommended Citation: Ramirez J, Bordon J, Cavallazzi R, et al. Characteristics and outcomes of adults hospitalized with SARS-CoV-2 community-acquired pneumonia in Louisville, Kentucky. Univ Louisville J Respir Infect 2020; 4(1):Article 72. doi: 10.18297/jri/vol4/iss1/72.

\begin{abstract}
Background: Patients infected with the novel coronavirus SARS-CoV-2 are frequently hospitalized with communityacquired pneumonia (CAP). The objective of this study was to define the clinical characteristics and outcomes of hospitalized patients with SARS-CoV-2 CAP in the city of Louisville, $\mathrm{KY}$.

Methods: This was a retrospective observational study of 700 patients with SARS-CoV-2 infection hospitalized at eight of the adult hospitals in the city of Louisville. Patients with 1) a positive RT-PCR for SARS-CoV-2, 2) fever, cough, or shortness of breath, and 3) an infiltrate on chest imaging were defined as having SARS-CoV-2 CAP. Demographic characteristics of the study population were compared with census data from the city of Louisville. For each patient, more than 500 variables were abstracted from electronic medical records and recorded using Research Electronic Data Capture software. Data were analyzed by descriptive and inferential statistics using $R$ version 3.4.0.
\end{abstract}

Results: SARS-CoV-2 CAP was identified in 632 (90\%) patients hospitalized with COVID-19. The median age of the patients was 63 years, $53 \%$ were females, $31 \%$ were black, and $12 \%$ were Hispanic. The most frequent comorbidities were hypertension (56\%), obesity (50\%), and diabetes (33\%). Mortality was $17 \%$ for the total population and $34 \%$ for the 249 patients admitted to ICU. For each category of race, ethnicity, or comorbidity, the proportion of hospitalized patients with SARS-CoV-2 CAP was significantly different compared to the Louisville population $(P<0.001)$.

Conclusion: Patients of black race, Hispanic ethnicity, and patients with a history of hypertension, obesity, or diabetes are overrepresented among hospitalized patients with SARSCoV-2 CAP compared to the Louisville population. Hospitalized patients with SARS-CoV-2 CAP are likely to require ICU care, with death occurring in approximately one of six hospitalizations.

\section{Introduction}

Coronavirus disease 2019 (COVID-19) is a multisystem disease produced by the novel coronavirus SARS-CoV2. COVID-19 manifests usually as an influenza-like syndrome due to multiplication of SARS-CoV-2, primarily in the epithelial cells of the upper respiratory tract. More severe disease occurs when the virus invades and multiplies in the lower respiratory tract particularly in alveolar epithelial type II cells.[1] At this stage the patient will present with a clinical syndrome of community-acquired pneumonia (CAP). SARS-CoV$2 \mathrm{CAP}$ is a common clinical presentation in hospi- talized patients with COVID-19. If the infection advances, viral replication accelerates, and the epithelialendothelial barrier integrity is compromised. At this next stage the patient may develop acute respiratory distress syndrome (ARDS) due to pulmonary edema filling the alveolar spaces with hyaline membrane formation.[1]

A significant amount of literature has been published describing the characteristics and outcomes of patients hospitalized with COVID-19, but clinical characteristics of patients with SARS-CoV-2 CAP, as well as their clinical outcomes, has been described in a limited num- 
ber of studies. Before the development of the COVID19 pandemic, we completed a population-based study of all hospitalized patients with CAP in the city of Louisville, KY. We prospectively enrolled all consecutive patients with CAP hospitalized to any of the adult hospitals in the city of Louisville during a period of 2 years.[2] Taking advantage of this well-established citywide clinical research network, we evaluated the first 700 hospitalized patients with a diagnosis of COVID19 with the objective to define the clinical characteristics and outcomes of hospitalized patients with SARSCoV-2 CAP in the city of Louisville.

\section{Methods}

\section{Study design, subjects, and setting}

This was a retrospective observational study of the first 700 patients with a diagnosis of SARS-CoV-2 hospitalized to any of the eight adult hospitals in the city of Louisville, KY. The study started on March 5 2020, when the first hospitalized patient with COVID-19 was identified in Louisville and ended on July 12020 . Patients' follow-up ceased at hospital discharge.

\section{Data collection}

Data were abstracted from hospital electronic medical records. Collected data included patient age, sex, race, ethnicity, body mass index, residence, medical and social history, physical examination findings, laboratory findings, chest radiographs and chest CT findings, medications, intensive care unit (ICU) admission, and need for mechanical ventilation. Race was categorized as black, white, and other. Ethnic group was categorized as Hispanic or non-Hispanic. Race and ethnicity were treated independently for analysis.

\section{Study definitions}

SARS-CoV-2 Infection: A patient hospitalized with a positive SARS-CoV-2 reverse transcriptase polymerase chain reaction (RT-PCR) from a nasopharyngeal swab or other respiratory sample.

SARS-CoV-2 CAP: A patient hospitalized with SARSCoV-2 infection with fever, cough, or shortness of breath, and with evidence of pulmonary infiltrates at chest radiograph or chest computer tomography scan (CT-scan).

SARS-CoV-2 CAP with ARDS: A patient with SARSCoV-2 CAP with evidence of acute respiratory distress syndrome (ARDS) define as bilateral opacities on a chest radiograph or computed tomographic (CT) scan not fully explained by cardiac failure or fluid overload with $\mathrm{PaO} 2 / \mathrm{FiO} 2 \leq 300 \mathrm{mmHg}$, on ventilator settings that include positive end-expiratory pressure (PEEP) $\geq 5 \mathrm{~cm} \mathrm{H} 2 \mathrm{O}$.[3]

\section{Clinical presentation}

The National Institutes of Health (NIH) guidelines for COVID-19 classified adults with SARS-CoV-2 infection in several severity of illness categories.[4] Using the $\mathrm{NIH}$ model, we classified the clinical presentation of hospitalized patients with COVID-19 in 9 stages. The following definitions were used:

Stage 1: SARS-CoV-2 Asymptomatic Infection. A patient with positive SARS-CoV-2 RT-PCR and no COVID-19 related symptoms.

Stage 2: SARS-CoV-2 Mild Infection. A patient with positive SARS-CoV-2 RT-PCR and COVID-19 related symptoms but without evidence of pulmonary infiltrates at chest imaging (e.g. no evidence of CAP).

Stage 3: SARS-CoV-2 CAP with no supplemental oxygen. A patient with SARS-CoV-2 CAP not receiving supplemental oxygen.

Stage 4: SARS-CoV-2 CAP and low-flow supplemental oxygen. A patient with SARS-CoV-2 CAP receiving supplemental oxygen delivered by a low-flow system (e.g. nasal cannula).

Stage 5: SARS-CoV-2 CAP and high-flow supplemental oxygen or non-invasive ventilation. A patient with SARS-CoV-2 CAP receiving supplemental oxygen delivered by a high-flow system (e.g. high-flow nasal cannula) or non-invasive ventilation.

Stage 6: SARS-CoV-2 CAP and mechanical ventilation . A patient with SARS-CoV-2 CAP intubated receiving invasive mechanical ventilation.

Stage 7: SARS-CoV-2 CAP and Mild ARDS. A patient with SARS-CoV-2 CAP on mechanical ventilation and development of ARDS with a $\mathrm{PaO} 2 / \mathrm{FiO} 2>200 \mathrm{mmHg}$, but $\leq 300 \mathrm{mmHg}$.

Stage 8: SARS-CoV-2 CAP and Moderate ARDS. A patient with SARS-CoV-2 CAP on mechanical ventilation and development of ARDS with a $\mathrm{PaO} 2 / \mathrm{FiO} 2$ $>100 \mathrm{mmHg}$, but $\leq 200 \mathrm{mmHg}$.

Stage 9: SARS-CoV-2 CAP and Severe ARDS. A patient with SARS-CoV-2 CAP on mechanical ventilation and development of ARDS with a PaO2/FiO2 is $\leq 100$ $\mathrm{mmHg}$.

\section{Severity of disease and mortality prediction}

Severity of SARS-CoV-2 CAP at time of hospitalization and mortality prediction was evaluated using two well established CAP scores, the pneumonia severity index (PSI) score and the CURB-65 score.[5,6]. 


\section{SARS-CoV-2 RNA levels}

Relative SARS-CoV-2 RNA levels at time of hospitalization were estimated using the number of cycles in the RT-PCR assay needed to amplify viral RNA to reach a detectable level (cycle threshold). Levels of relative SARS-CoV-2 RNA were classified as low, intermediate and high.

\section{Geospatial epidemiology}

The geomasked location of the home address of each patient with SARS-CoV-2 CAP who was enrolled in the study was obtained through the US Census Bureau website.[7] A kernel density heatmap was created using each patient's address at the time of SARSCoV-2 CAP hospitalization. Areas of elevated relative risk of contracting SARS-CoV-2 CAP based on underlying population density were identified using Kuldorff's spatial scan statistic.

\section{Cardiac and cardiovascular events}

The following cardiac or cardiovascular events that were present at the time of admission or developed during hospitalization were collected: development of heart failure, cardiac arrest, cardiogenic shock, acute myocardial infarction, pulmonary edema, new arrhythmia, acute worsening of a chronic arrhythmia, cerebrovascular accident, pulmonary embolism, myocarditis, and deep vein thrombosis.

\section{Co-infections}

A patient with an isolated micro-organism from blood, respiratory, or urine samples was defined as having a co-infection. Community-acquired co-infections were defined as identification of micro-organisms during the first 3 days of hospital admission. Hospital-acquired co-infections were defined as micro-organisms identified more than 3 days after hospital admission.

\section{Clinical outcomes}

Patients who were discharged alive were considered as clinical successes and patients who died during hospitalization were considered as clinical failures. Binary outcomes evaluated included need for invasive mechanical ventilation, admission to ICU, septic shock, ARDS, and death. Time-to-event outcomes evaluated included time to hospital discharge, time to initiation of mechanical ventilation, time to ICU admission, time to septic shock, time to ARDS, and time to death.

\section{Statistical analysis}

Continuous patient characteristics were summarized as medians and interquartile ranges and categorical patient characteristics were summarized as frequencies and percentages. Comparisons to Louisville demo- graphics were performed using one-sample t-tests of proportions, with null values equal to the Louisville proportion, taken from the 2017 Behavioral Risk Factor Surveillance System (BRFSS) Smart Data database and the 2019 American Community Survey 5-year estimates. [8,9] Analyses were performed with $R$ version 3.4.0. $P$-values were 2 -sided, with statistical significance set at $P<0.05$.

\section{Study coordinating center}

The Center of Excellence for Research in Infectious Diseases (CERID) located at the University of Louisville Division of Infectious Diseases, directed all operations of the study (CERIDLouisville.org). Members of CERID developed the study data collection form and the study database, collected data from hospital electronic medical records and recorded data into the study database. Data were collected and managed using REDCap electronic data capture tools hosted at the University of Louisville. REDCap (Research Electronic Data Capture) is a secure, web-based software platform designed to support data capture for research studies, providing an intuitive interface for validated data capture.[10] The CERID data quality team performed quality control of collected data. After all data queries were resolved, the study database was locked for data analysis.

\section{Human subjects protection}

The study was approved by the Institutional Review Board (IRB) at the University of Louisville Human Subjects Research Protection Program Office (IRB number 20.0257) and by the research offices at each participating hospital. The study was exempt from informed consent.

\section{Results}

\section{Study population and clinical presentation}

From a total of 700 patients hospitalized with COVID19 , a total of 632 patients $(90 \%)$ were hospitalized with SARS-CoV-2 CAP. A bar chart describing the patients' clinical presentation at time of hospitalization is depicted in Figure 1. The most common clinical presentation was SARS-CoV-2 CAP in patients not receiving supplemental oxygen (stage 3). The primary clinical diagnoses at time of hospitalization for 68 patients in stages 1 and 2 (patients without SARS-CoV-2 CAP) included labor, trauma, and urinary tract infections. Demographics and comorbidities for hospitalized patients with SARS-CoV-2 CAP are depicted in Table 1.

Comparisons of SARS-CoV-2 CAP patients' demographics to the Louisville population are shown in Figure 2. Comparisons of SARS-CoV-2 CAP patients' 


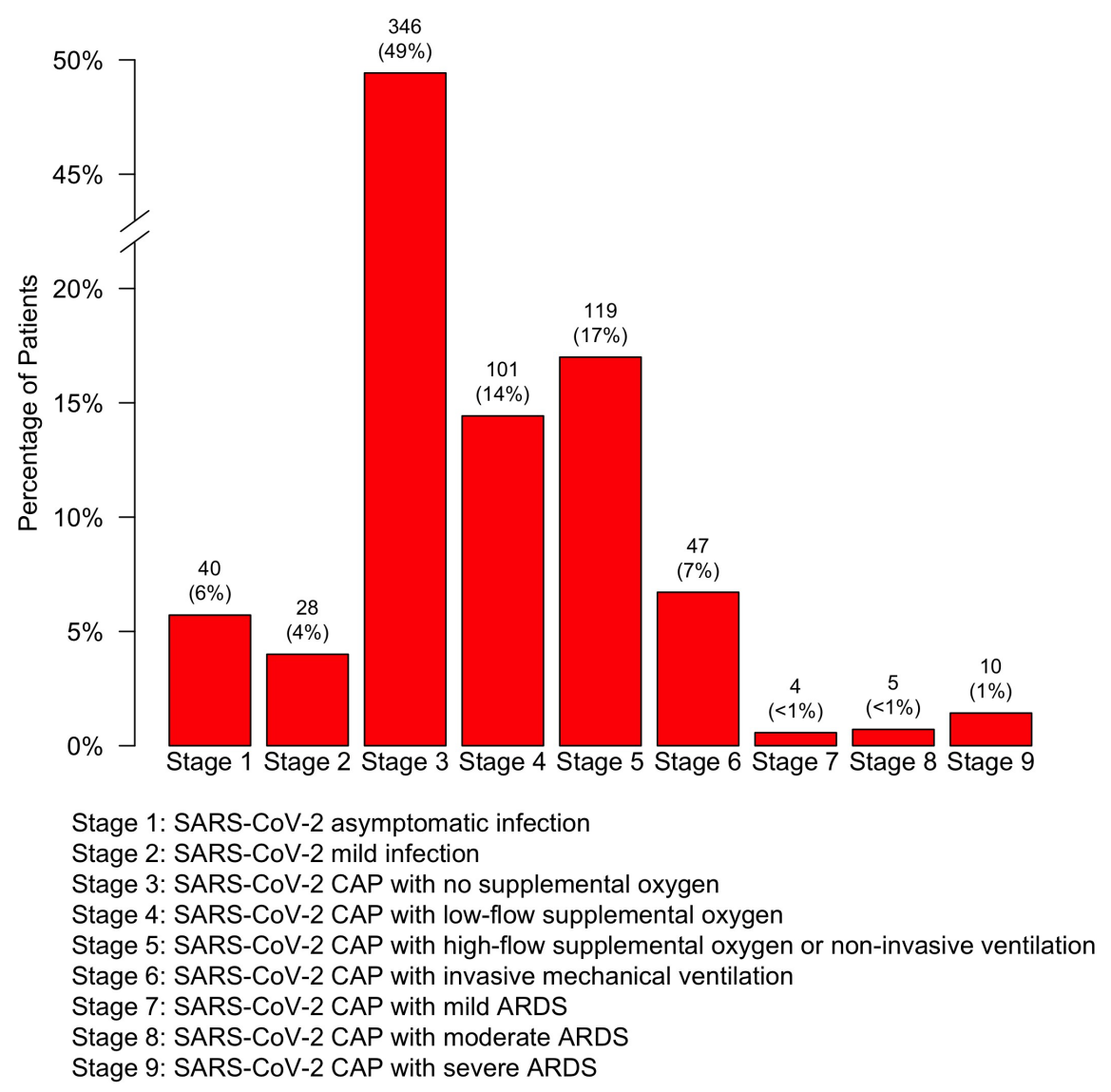

Figure 1. Study bar chart indicating the number of COVID-19 hospitalized patients in each one of the nine stages of clinical presentation.

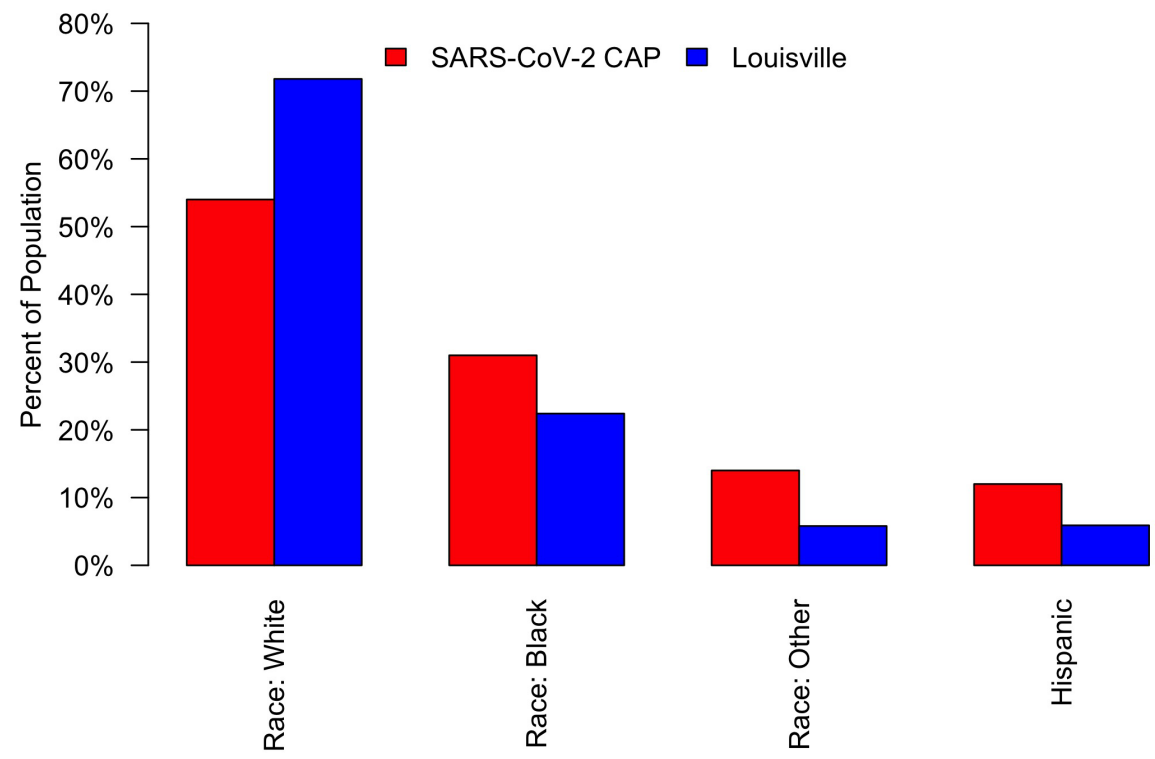

Figure 2. Comparison of patient demographics to those of the city of Louisville. All data are reported as percentage of the population in the demographic. 
Table 1. Patient demographics and medical history.

\begin{tabular}{lc}
\hline Characteristic & $n(\%)^{*}$ \\
\hline Total patients & 632 \\
Age (median [IQR]) & $63[48,74]$ \\
Sex: Male & $294(47)$ \\
Race & \\
$\quad$ Black & $198(31)$ \\
White & $343(54)$ \\
Other & $90(14)$ \\
Hispanic & $74(12)$ \\
Smoking history & \\
$\quad$ Never & $404(64)$ \\
Current & $58(9)$ \\
Former & $170(27)$ \\
Hypertension & $352(56)$ \\
Obesity & $315(50)$ \\
Diabetes & $214(33)$ \\
Renal disease & $119(19)$ \\
Heart failure & $99(16)$ \\
Coronary artery disease & $95(15)$ \\
COPD & $96(15)$ \\
Cerebrovascular disease & $86(14)$ \\
Asthma & $67(11)$ \\
Obstructive sleep apnea & $60(9)$ \\
Neoplastic disease (active or within the last year) & $45(7)$ \\
Other immunocompromising condition or therapies & $29(5)$ \\
Liver disease (non-cirrhotic) & $21(3)$ \\
Cirrhosis & $8(1)$ \\
\hline Abbreviations: COPD & \\
\hline
\end{tabular}

Abbreviations: COPD, chronic obstructive pulmonary disorder; $I Q R$, interquartile range.

* Except where otherwise specified.

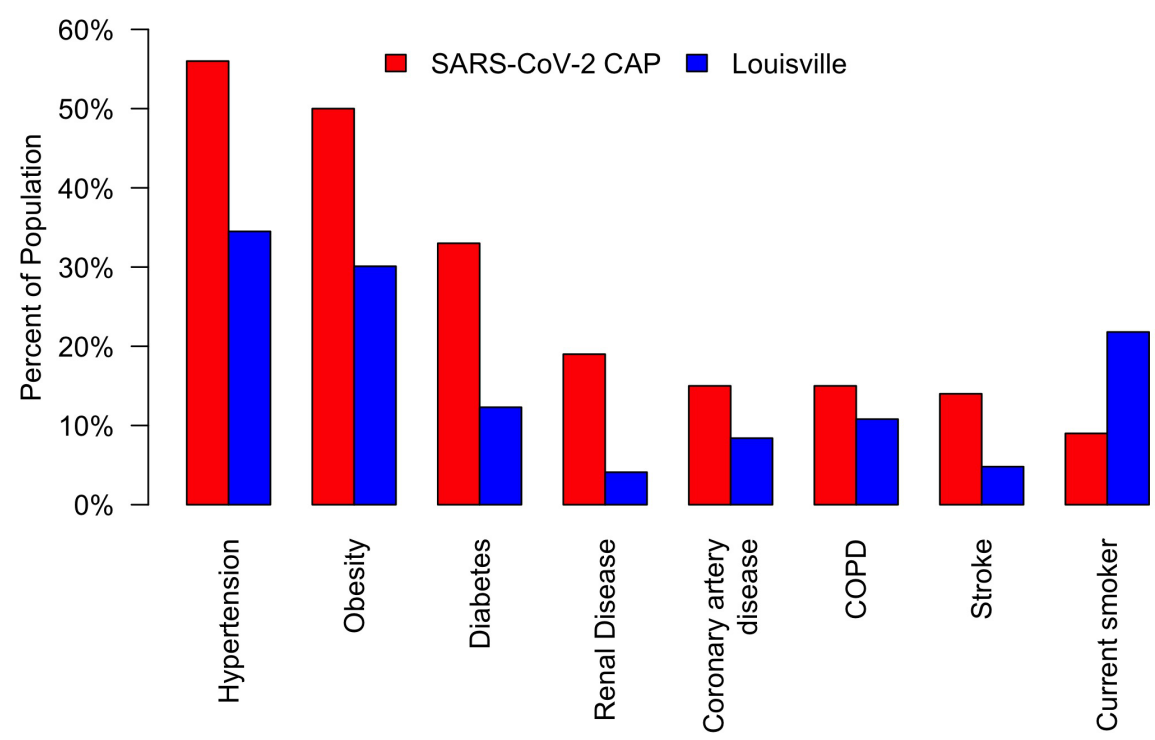

Figure 3. Comparison of patient comorbidities to those of the city of Louisville. All data are reported as percentage of the population with the comorbidity. 


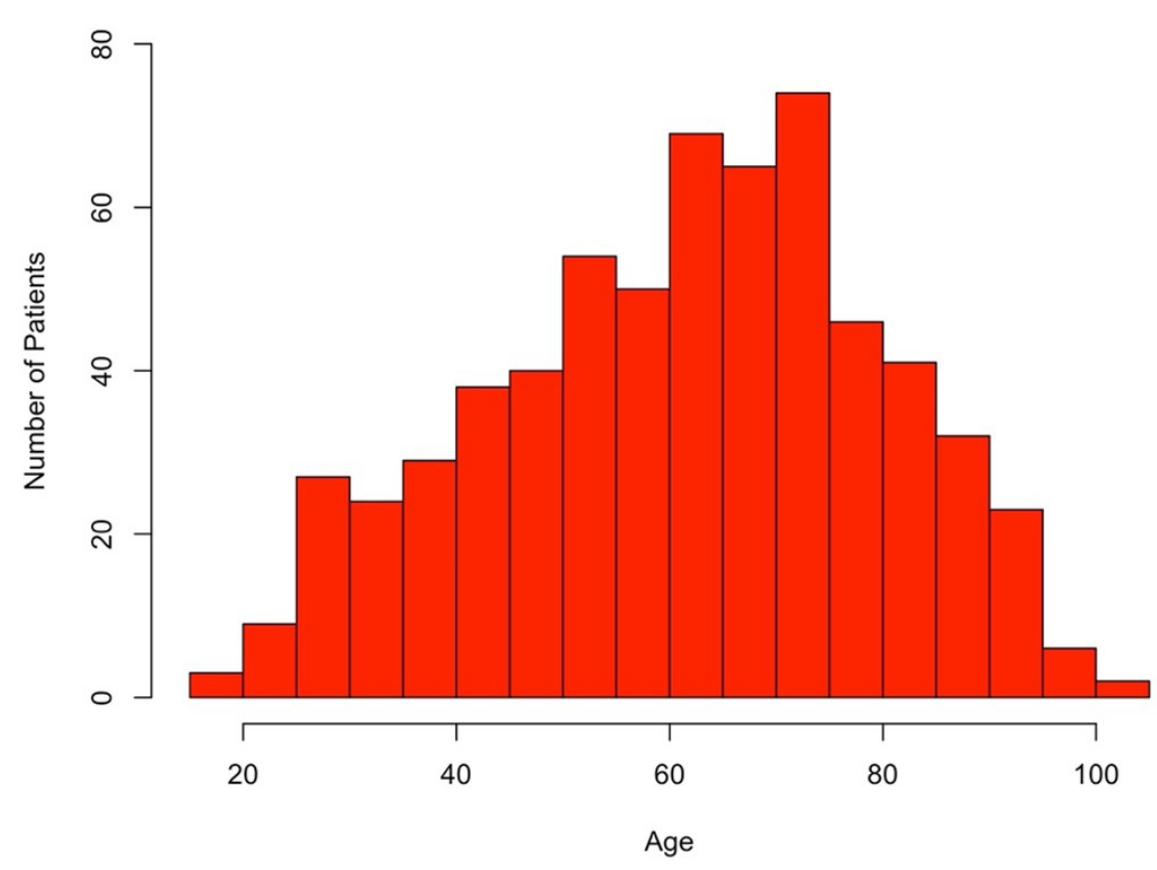

Figure 4. Histogram depicting the number of patients with SARS-CoV-2 CAP per age group.

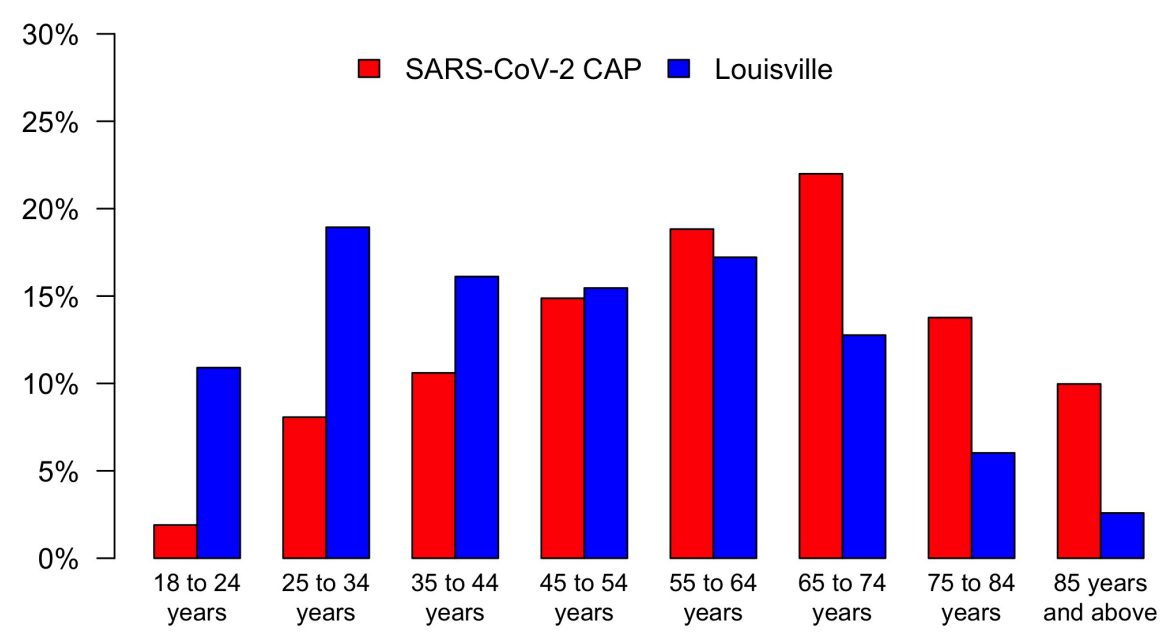

Figure 5. Comparison of percentage distribution of SARS-CoV-2 CAP patient age to the Louisville population (left) patients hospitalized with SARS-CoV-2 CAP, (right) the adult population of Louisville. 
Table 2. Signs and symptoms for patient hospitalized with SARS-CoV-2 CAP.

\begin{tabular}{lc}
\hline Sign/symptom & $n(\%)$ \\
\hline Total patients & 632 \\
Fever or subjective fever & $436(69)$ \\
Cough & $425(67)$ \\
Dyspnea (shortness of breath) & $407(64)$ \\
Fatigue & $218(34)$ \\
Muscle ache (myalgia) & $160(25)$ \\
Nausea & $139(22)$ \\
Diarrhea & $114(18)$ \\
Sputum production & $95(15)$ \\
Vomiting & $79(12)$ \\
Chest pain/tightness & $78(12)$ \\
Confusion & $71(11)$ \\
Headache & $71(11)$ \\
Sore throat & $52(8)$ \\
Nasal congestion & $52(8)$ \\
Dizziness & $42(7)$ \\
Rhinorrhea (runny nose) & $21(3)$ \\
Anosmia & $15(2)$ \\
Agusia & $15(2)$ \\
Hemoptysis (blood in sputum) & $7(1)$ \\
Constipation & $4(1)$ \\
\hline
\end{tabular}

Table 3. Vital signs and laboratory values. The third column describes the number of patients for whom data were available.

\begin{tabular}{|c|c|c|}
\hline Variable & Median [IQR] & $n$ \\
\hline Heart rate (beats/min) & $96.5[82.0,111.0]$ & 632 \\
\hline Respiratory rate (breaths/min) & $22.0[18.0,27.0]$ & 632 \\
\hline Systolic blood pressure (mmHg) & $121.0[106.0,140.2]$ & 632 \\
\hline Diastolic blood pressure (mmHg) & $67.5[57.0,80.0]$ & 632 \\
\hline Temperature $\left({ }^{\circ} \mathrm{C}\right)$ & $37.7[37.0,38.7]$ & 632 \\
\hline $\mathrm{SpO}_{2} / \mathrm{FiO}_{2}$ & $428.6[290.6,452.4]$ & 631 \\
\hline $\mathrm{PaO}_{2} / \mathrm{FiO}_{2}$ & $187.2[99.2,297.7]$ & 238 \\
\hline WBC $\times 1,000$ per uL & $6.3[4.6,8.8]$ & 627 \\
\hline Neutrophils $\times 1,000$ per uL & $4.6[3.0,7.4]$ & 610 \\
\hline Lymphocytes $\times 1,000$ per uL & $1.0[0.7,1.4]$ & 615 \\
\hline Neutrophil/lymphocyte & $4.5[2.7,7.8]$ & 609 \\
\hline Hematocrit (\%) & $38.8[35.2,42.4]$ & 627 \\
\hline Glucose (mg/dL) & $123.0[104.0,166.0]$ & 629 \\
\hline Blood urea nitrogen (mg/dL) & $17.0[11.0,28.2]$ & 626 \\
\hline Creatinine $(\mathrm{mg} / \mathrm{dL})$ & $1.0[0.7,1.4]$ & 627 \\
\hline Ferritin (ng/dL) & $398.0[176.0,891.0]$ & 435 \\
\hline Procalcitonin (ug/L) & $0.1[0.1,0.4]$ & 504 \\
\hline Lactate $(\mathrm{mmol} / \mathrm{L})$ & $1.3[1.1,1.8]$ & 465 \\
\hline D-Dimer (ng/mL) & $736.0[388.5,1517.0]$ & 447 \\
\hline $\mathrm{IL}-6(\mathrm{pg} / \mathrm{mL})$ & $58.2[29.6,131.3]$ & 198 \\
\hline C-reactive protein (mg/L) & $79.3[38.0,170.1]$ & 436 \\
\hline
\end{tabular}

Abbreviations: IQR, interquartile range; WBC, white blood cell count. comorbidities to the Louisville population are shown in Figure 3. All comparisons between patient demographics and comorbidities to the Louisville population were statistically significant $(P<0.001)$. The age distribution showing the number of adults hospitalized with SARS-CoV-2 CAP is depicted in Figure 4. A comparison of the percentage distribution of age groups between adults hospitalized with SARS-CoV-2 CAP and the adult Louisville population is shown in Figure 5.

Signs and symptoms in patients with SARS-CoV-2 CAP are depicted in Table 2. Median time from symptoms onset to hospitalization was 4 days.

Vital signs and laboratory values for patients hospitalized with SARS-CoV-2 CAP at admission are depicted in Table 3.

The characteristic chest CT-scans imaging of diffuse, peripheral ground-glass opacities were present in 324 of $439(74 \%)$ patients with chest CT performed.

Medications used in adults hospitalized with SARSCoV-2 CAP during hospitalization are depicted in Table 4 .

\section{Severity of disease and associated mortality}

The severity of CAP at time of hospitalization according to CURB-65 and PSI scores with associated mortality per class or score are depicted in Figure 6.

\section{SARS-CoV-2 RNA levels}

Cycle threshold (CT) values were available for a total 405 SARS-CoV-2 CAP patients. For these patients, the lowest CT value from the N1, N2, and N3 genes was used to represent the relative viral RNA levels, as a lower CT value indicates a high viral load. CT values are depicted in Figure 7.

\section{Geospatial epidemiology}

The kernel density heatmap created using each patient's home address at the time of hospitalization due to SARS-CoV-2 CAP is depicted in Figure 8.

\section{Cardiac and cardiovascular events}

A total of 120 patients (19\%) developed cardiac or cardiovascular events. The list of cardiac and cardiovascular events is depicted on Table 5.

\section{Co-infections}

A total of 67 SARS-CoV-2 CAP patients had coinfections. The list of patients with communityacquired $(n=25)$ and hospital-acquired $(n=42)$ coinfections is depicted on Table 6. For each type of coinfection, the culture sites were characterized. 
Table 4. Medications for treatment of SARS-CoV-2 CAP.

\begin{tabular}{lc}
\hline Medication & $n(\%)$ \\
\hline Total patients & 632 \\
Azithromycin & $398(63)$ \\
Low molecular weight heparin & $394(62)$ \\
Hydroxychloroquine & $239(38)$ \\
Steroid & $145(23)$ \\
Heparin & $114(19)$ \\
Convalescent plasma & $50(8)$ \\
Remdesivir & $31(5)$ \\
\hline
\end{tabular}

\section{Clinical outcomes}

From the total population of 632 patients hospitalized with SARS-CoV-2 CAP, clinical success (discharged alive) was documented in $527(83 \%)$ patients and clinical failure (died during hospitalization) in $105(17 \%)$ patients. The median length of hospital stay was 7 days. Time to hospital discharge and time to in-hospital death is depicted in Figure 9A and 9B. A total of 249 patients were admitted to the ICU, with 124 admitted on the first day. A total of 159 patients required mechanical ventilation. Mortality among patients admitted to the ICU was $34 \%$. Time to ICU admission and time to mechanical ventilation are depicted in Figure $9 \mathrm{C}$ and 9D. Over the course of hospitalization, a total of 85 patients with SARS-CoV-2 CAP developed septic shock and 98 patients developed ARDS. Time to septic shock and time to ARDS are depicted in Figure 9E and 9F. Mortality was much higher in comorbid patients, ranging from 16 to $36 \%$. Figure 10 depicts crude mortality by demographics and comorbidities.

\section{Discussion}

Our study indicates that SARS-CoV-2 CAP is present in $90 \%$ of all patients hospitalized with COVID-19. We previously described the clinical characteristics of hospitalized patients in the city of Louisville with CAP due to the four endemic serotypes of coronaviruses.[11] The most frequent serotype causing CAP was CoV-OC43, followed by CoV-229E, CoV-HKU1, and CoV-NL63. At this time, SARS-CoV-2 has emerged as the predominant coronavirus causing CAP. Hospitalized COVID-19 patients without CAP constitute a heterogenous population. The most common admission diagnoses in patients without CAP included labor \& delivery and trauma. In the majority of these patients COVID-19 was present as an asymptomatic infection.

Among the patients hospitalized with SARS-CoV-2 in
Table 5. Cardiac and cardiovascular events in patients hospitalized due to SARS-CoV-2 CAP.

\begin{tabular}{lc}
\hline Variable & $n(\%)$ \\
\hline Total patients & 632 \\
New arrhythmia & $52(8)$ \\
Heart failure & $25(4)$ \\
Cardiac arrest & $20(3)$ \\
Acute worsening of chronic arrhythmia & $20(3)$ \\
Cardiogenic shock & $15(2)$ \\
Acute myocardial infarction & $13(2)$ \\
Pulmonary edema & $13(2)$ \\
Cerebrovascular accident & $7(1)$ \\
Pulmonary embolism & $6(1)$ \\
Deep vein thrombosis & $6(1)$ \\
Myocarditis & $2(<1)$ \\
\hline
\end{tabular}

the city of Louisville, adults of black race or Hispanic ethnicity are overrepresented in relation to the census population of the city. Our study supports data from other areas of the Unites States indicating a higher percentage of black patients hospitalized with COVID19.[12]

Hypertension was the most frequent comorbidity in hospitalized patients with SARS-CoV-2 CAP in Louisville. Patients with hypertension, as well as patients with obesity, diabetes, renal disease, coronary artery disease, COPD, and cerebrovascular disease were overrepresented in the population hospitalized with SARS-CoV-2 CAP when compared to the general Louisville population. Hypertension was also reported as the most common comorbidity in hospitalized patients in a large CDC multicenter study.[13]

A clinical syndrome of CAP was the predominant clinical presentation of COVID-19 in hospitalized patients. The most common signs and symptoms in our population were fever, cough, and shortness of breath. These are the most frequent signs and symptoms for hospitalized patients with COVID-19 in the US.[13] Some of our patients presented with a flu-like clinical syndrome characterized by fever, sore throat, fatigue and muscle aches. During the next winter season, the flu-like illnesses produced by SARS-CoV-2 will need to be added to the differential diagnosis of flu-like illness produced by other winter respiratory viruses. Surveillance data on circulating respiratory viruses in the community will be important to facilitate the clinical management of patients with flu-like illness this winter. SARS-CoV-2 started to circulate in the Louisville community at the end of the 2019-2020 winter season. Coincidental to the emergence of SARS-CoV-2 in Louisville, we documented a significant decrease in the circulation of other serotypes of coronavirus, as well as a decrease in respiratory syncytial virus, rhinovirus, and influenza.[14] The decrease in common respiratory viruses at the end of last winter was most likely related to the different 


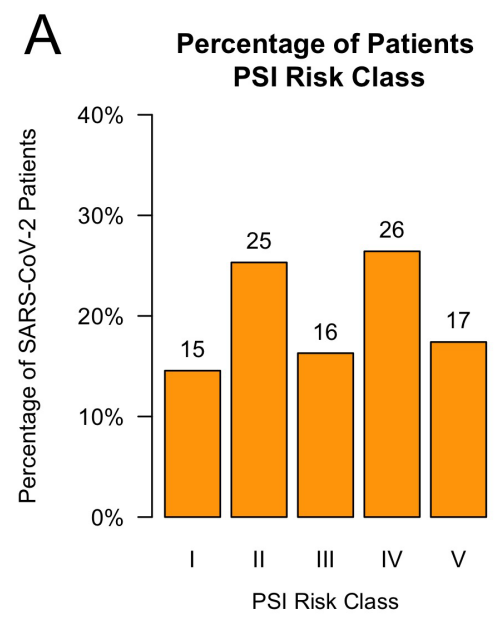

C Percentage of Expired Patients PSI Risk Class

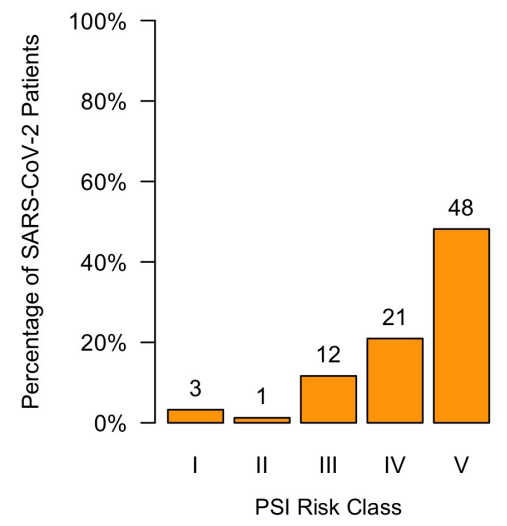

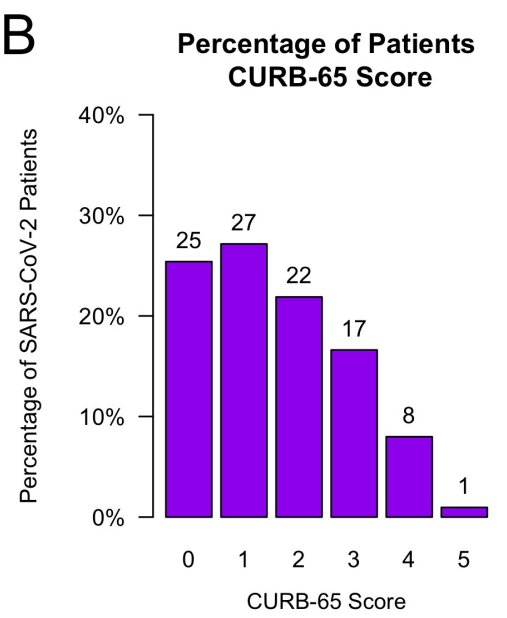
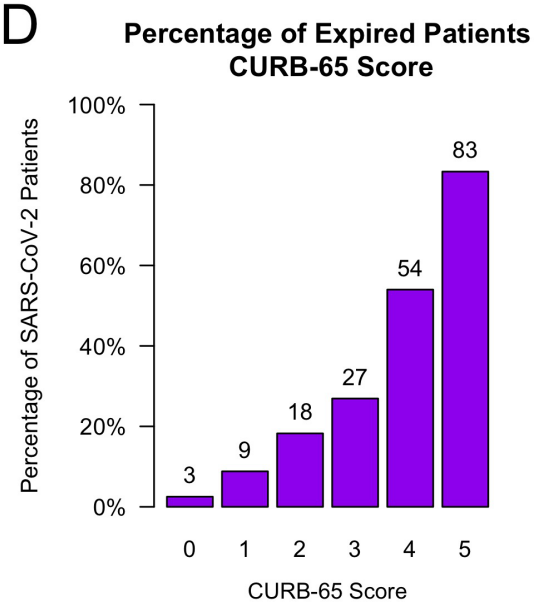

Figure 6. The distribution of patients hospitalized with SARS-CoV-2 CAP in PSI risk classes (top-left) and the distribution of patients hospitalized with SARS-CoV-2 CAP in CURB-65 scores (top-right) are shown in the top of the figure. The associated percentage of in-hospital mortality by PSI risk class (bottom-left) and by CURB-65 score (bottom-right) are shown below.

steps implemented in the city related to social distancing, primarily, the closure of non-essential businesses.

Severity of CAP at time of hospitalization is usually evaluated using the PSI score or the CURB-65 score. When we applied these scores to our population of hospitalized patients with SARS-CoV-2 CAP, the CURB-65 score better discriminated risk of death during hospitalization, from $3 \%$ risk for death in patients with a score of 0 , to $70 \%$ risk for death for patients with a score of 5. Physicians may use the CURB-65 to supplement clinical judgment when evaluating severity and risk of death in patients with SARS-CoV-2 CAP. One weakness of our study is the lack of data regarding ambulatory patients with COVID-19 in the city of Louisville, therefore we cannot evaluate the role of severity scores in deciding ambulatory care.

On the first day of hospitalization, need for ICU care was documented in $20 \%$ of our SARS-CoV-2 CAP population. A high proportion of patients hospitalized with COVID-19 requiring initial high level of care was also documented in a large cohort study in the United Kingdom.[15] Admission to the ICU during the first day of hospitalization may indicate a rapid progression of SARS-CoV-2 infection in some patients or may indicate that some patients may not look for early medical attention. The initial treatment strategies from the time of study were not evidence-based, as there was a lack of studies from the corresponding study period. It is also important to note that several patients hospitalized with SARS-CoV-2 CAP were treated with hydroxychloroquine. This medication is not currently recommended for hospitalized patients, but a series of patients hospitalized at the beginning of the pandemic were treated with hydroxychloroquine even though the evidence for its efficacy was lacking.

Using the initial cycle threshold (Ct) levels, we estimated the SARS-CoV-2 viral load at the time of hospitalization in 405 patients with SARS-CoV-2 CAP. Ct val- 


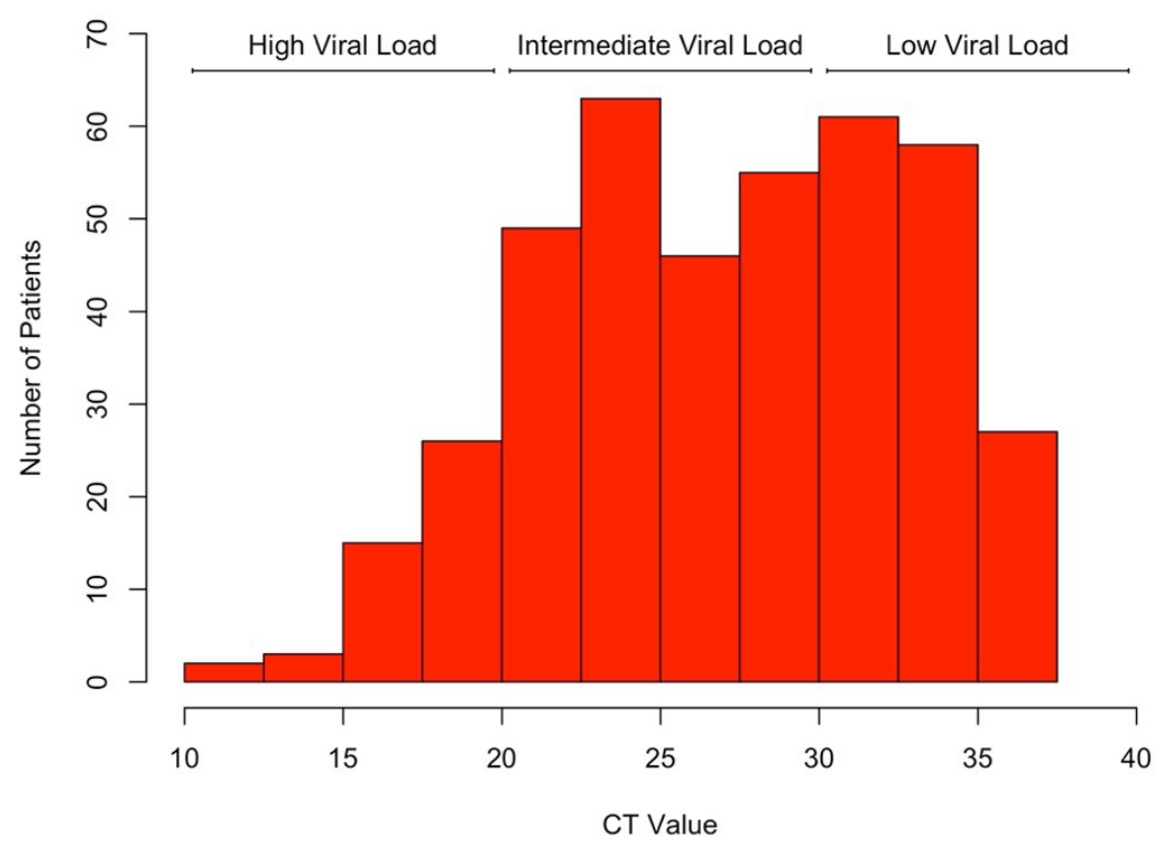

Figure 7. Distribution of the lowest CT value from the N1, N2, and N3 gene. Lower CT values are analogous to higher viral loads. Level of viral load was classified into high, intermediate, and low.

SARS-CoV-2 CAP Cases in Louisville, KY

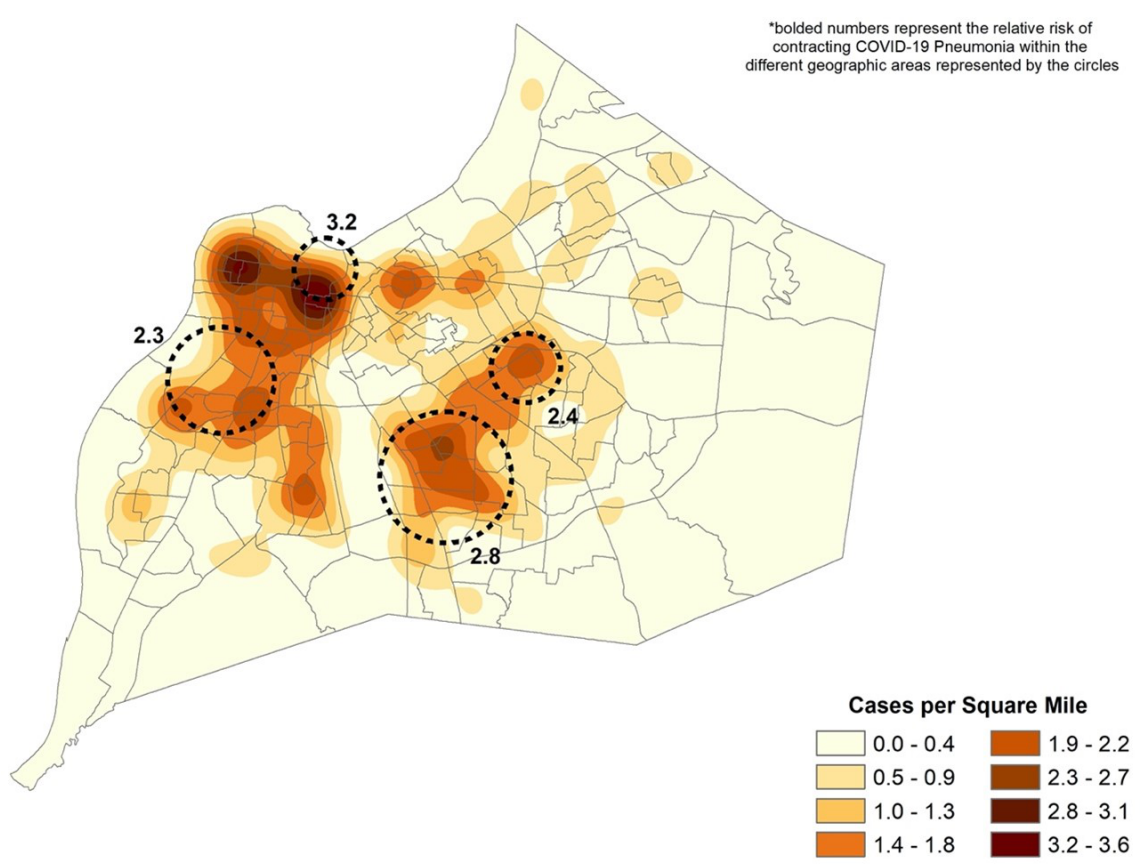

Figure 8. Kernel density heatmap of residential addresses of patients hospitalized with SARS-CoV-2 CAP. Four distinct areas of elevated risk are identified in the dashed circles. 

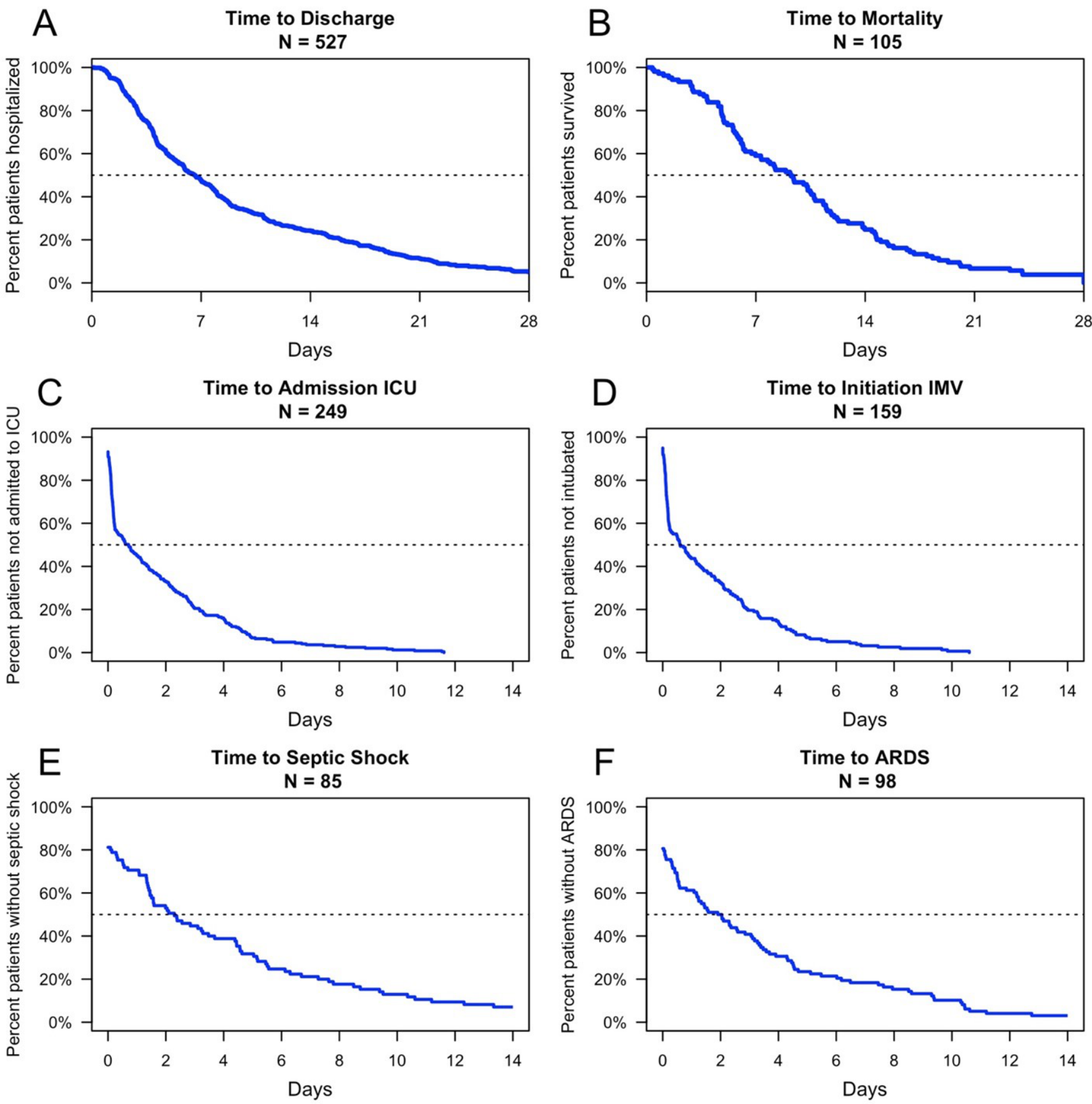

Figure 9. Time to event outcomes, in days. A) Time to discharge from admission, B) time to mortality from admission, C) time to ICU admission, D) time to initiation of IMV, E) time to septic shock, F) time to ARDS. 
Table 6. Number of patients with coinfections, micro-organisms, and culture sites in adults hospitalized with SARS-CoV-2 CAP.

\begin{tabular}{|c|c|c|}
\hline Organism & Patients $(n)$ & Culture site $(n)$ \\
\hline $\begin{array}{l}\text { Community-acquired coinfections } \\
\text { Escherichia coli } \\
\text { Klebsiella pneumoniae } \\
\text { Haemophilus influenzae } \\
\text { Streptococcus pneumoniae } \\
\text { Acinetobacter spp. } \\
\text { Enterobacter spp. } \\
\text { Influenza B } \\
\text { Moraxella catarrhalis } \\
\text { Parainfluenza virus } \\
\text { Pseudomonas aeruginosa } \\
\text { Respiratory syncytial virus } \\
\text { MSSA } \\
\text { Streptococcus bovis } \\
\text { Streptococcus pyogenes } \\
\text { Candida spp. }\end{array}$ & $\begin{array}{l}7 \\
3 \\
2 \\
2 \\
1 \\
1 \\
1 \\
1 \\
1 \\
1 \\
1 \\
1 \\
1 \\
1 \\
1\end{array}$ & $\begin{array}{l}\text { Blood (2), urine (5) } \\
\text { Sputum (1), urine (2) } \\
\text { Sputum (2) } \\
\text { Sputum (2) } \\
\text { Sputum (1) } \\
\text { Urine (1) } \\
\text { BAL (1) } \\
\text { Sputum (1) } \\
\text { BAL (1) } \\
\text { Urine (1) } \\
\text { BAL (1) } \\
\text { Sputum (1) } \\
\text { Blood (1) } \\
\text { Blood (1) } \\
\text { Blood (1) }\end{array}$ \\
\hline $\begin{array}{l}\text { Hospital-acquired coinfections } \\
\text { MRSA } \\
\text { Enterobacter spp. } \\
\text { Klebsiella pneumoniae } \\
\text { Pseudomonas aeruginosa } \\
\text { Escherichia coli } \\
\text { Stenotrophomonas spp. } \\
\text { Citrobacter spp. } \\
\text { Serratia spp. } \\
\text { Candida spp. } \\
\text { Aspergillus spp. } \\
\text { Coronavirus OC43 } \\
\text { MSSA }\end{array}$ & $\begin{array}{l}9 \\
5 \\
5 \\
5 \\
4 \\
4 \\
2 \\
2 \\
2 \\
2 \\
1 \\
1\end{array}$ & $\begin{array}{r}\text { Blood (1), sputum (7), Urine culture (1) } \\
\text { Blood (2), sputum (3) } \\
\text { Sputum (3), BAL (1), urine culture (1) } \\
\text { Blood (1), sputum (2), BAL (1), Urine (1) } \\
\text { Sputum (1), urine (3) } \\
\text { Sputum (3), BAL (1) } \\
\text { Sputum (1), BAL (1) } \\
\text { Sputum (1), urine (1) } \\
\text { Blood (1), urine (1) } \\
\text { Sputum (2) } \\
\text { NP swab (1) } \\
\text { Sputum (1) }\end{array}$ \\
\hline
\end{tabular}

Abbreviations: BAL, bronchoalveolar lavage; MRSA, methicillin-resistant Staphylococcus aureus; MSSA, methicillin-susceptible Staphylococcus aureus; NP, nasopharyngeal.

ues are inversely proportional to the amount of the target nucleic acid in the sample.[16] We identified some patients with high $\mathrm{Ct}$ values, indicating very low viral loads at time of hospitalization. It has been suggested that the concentration of viral RNA in throat swabs may be already on the decline for some patients at the time of initial presentation.[17]

The heatmap of patients hospitalized with SARS-CoV2 in the city of Louisville identifies areas of high relative risk that are very similar to areas that we previously identified as high risk for hospitalization due to CAP.[2] The clustering of hospitalized patients with CAP as well as SARS-CoV-2 CAP in the western section of the city is associated with census tracts where the average population has low annual income and is of predominantly black race.

Cardiovascular events at time of hospitalization or during hospitalization were documented in 19\% of hospitalized patients with SARS-CoV-2 CAP. New arrhythmias were the most frequent cardiovascular event, taking place in $8 \%$ of the patients hospitalized with SARSCoV-2 CAP. Cardiovascular complications may be due to increased cardiac demand produced by the infection, destabilization of vascular plaques due to systemic inflammation, hypercoagulability due to endothelial damage, or direct heart damage due to SARS-CoV2.[18]

A systematic review identified co-infections in $7 \%$ of hospitalized COVID-19 patients.[19] We identified co-infections in $8 \%$ of our SARS-CoV-2 CAP cohort; 25 were community-acquired and 42 were hospitalacquired. Viral respiratory infections such as influenza can predispose patients to co-infections of the respiratory tract. SARS-CoV-2 seems to predispose patients to infections in the respiratory tract but also outside of the respiratory tract. As an example, our group reported a case of a hospitalized patient with SARS-CoV-2 CAP and concomitant Escherichia coli bacteremia due to a prostatic abscess.[20]

Proper assessment of clinical outcomes is a crucial component of current research efforts in the field of COVID19. Studies of therapeutic interventions that evaluate clinical outcomes for all hospitalized patients with COVID-19 may be biased by the heterogenous popu- 


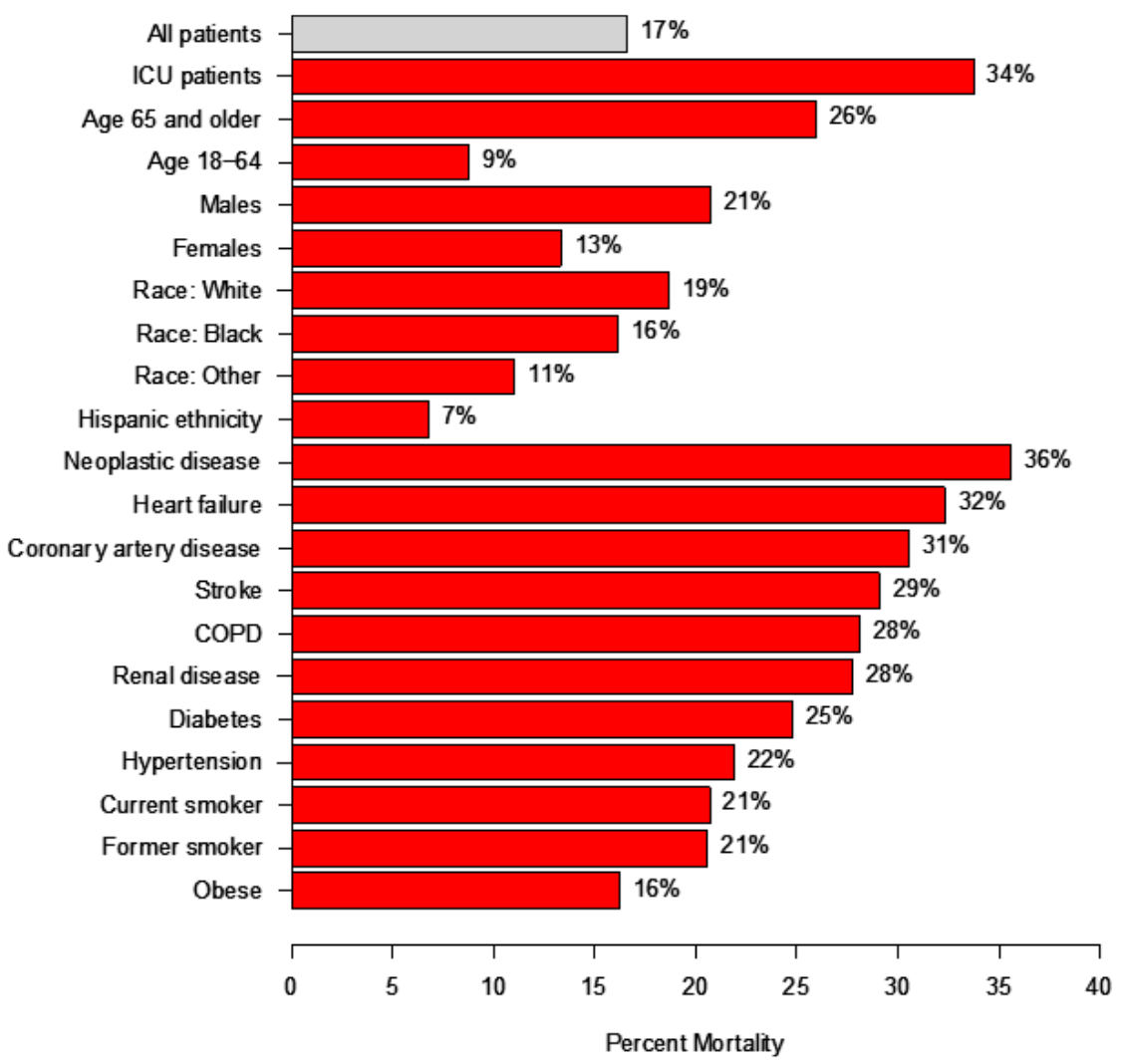

Figure 10. Mortality by demographics and comorbid conditions. The percentage mortality in each group is displayed.

lation that is admitted to the hospital with the general diagnosis of COVID-19. In our study of 632 hospitalized patients with SARS-CoV-2 and a clinical diagnosis of CAP, we evaluated five binary outcomes and six time-to-event outcomes. The mean length of stay was 7 days, with an in-hospital mortality of $17 \%$. For patients in the ICU mortality was 34\%. The ICU mortality in our cohort is higher than the mortality of $26 \%$ reported in a study of patients hospitalized to ICUs at 72 hospitals in Italy.[21]

In conclusion, SARS-CoV-2 CAP is the primary reason for hospitalization of patients with COVID-19. Patients with SARS-CoV-2 CAP account for 90\% of COVID-19 hospitalizations with an in-hospital mortality of $17 \%$. Community members who are elderly, of black race, Hispanic ethnicity, and having medical comorbidities are at high risk for hospitalization. The primary medical comorbidities in our population were hypertension, obesity, diabetes, renal disease and cerebrovascular disease. Future research in hospitalized patients with COVID-19 should concentrate primarily on the development of new management and treatment strategies for patients with SARS-CoV-2 CAP.
Received: October 29, 2020

Accepted: November 16, 2020

Published: December 7, 2020

Copyright: (C) 2022 The author(s). This original article is brought to you for free and open access by ThinkIR: The University of Louisville's Institutional Repository. For more information, please contact thinkir@louisville.edu. This article is distributed under the terms of the Creative Commons Attribution 4.0 International License (CC BY 4.0), which permits unrestricted use, distribution, and reproduction in any medium, provided the original author and source are credited.

Funding Source: The author(s) received no specific funding for this work.

Conflict of Interest: All authors declared no conflict of interest in relation to the main objective of this work. 


\section{References}

1. Wiersinga WJ, Rhodes $A$, Cheng AC, Peacock SJ, Prescott HC. Pathophysiology, transmission, diagnosis, and treatment of coronavirus disease 2019 (COVID-19): A review. Jama 2020; 324(8):782-93. doi: 10.1001/jama.2020.12839. PMID: 32648899 .

2. Ramirez JA, Wiemken TL, Peyrani $P$, et al. Adults hospitalized with pneumonia in the United States: Incidence, epidemiology, and mortality. Clin Infect Dis 2017; 65(11):180612. doi: 10.1093/cid/cix647. PMID: 29020164.

3. Ranieri VM, Rubenfeld GD, Thompson BT, et al. Acute respiratory distress syndrome: The berlin definition. JAMA 2012; 307(23):2526-33. doi: 10.1001/jama.2012.5669. PMID: 22797452.

4. National Institutes of Health. Clinical presentation of people with SARS-CoV-2 infection. Available at: https://www.covid19treatmentguidelines.nih.gov/ overview/clinical-presentation/. Accessed 20 October 2020.

5. Fine MJ, Auble TE, Yealy DM, et al. A prediction rule to identify low-risk patients with community-acquired pneumonia. N Engl J Med 1997; 336(4):243-50. doi: 10.1056/nejm199701233360402. PMID: 8995086.

6. Lim WS, van der Eerden MM, Laing R, et al. Defining community acquired pneumonia severity on presentation to hospital: An international derivation and validation study. Tho$\operatorname{rax} 2003$; 58(5):377-82. doi: 10.1136/thorax.58.5.377. PMID: 12728155

7. United States Census Bureau. Welcome to Geocoder. Available at: https://geocoding.geo.census.gov/geocoder/. Accessed 17 September 2020.

8. Centers for Disease Control and Prevention. Behavioral Risk Factor Surveillance System. Available at: https://www. cdc.gov/brfss/index.html. Accessed 17 November 2020.

9. United States Census Bureau. American Community Survey 2014-2018 5-year estimates now available. Online, 2019.

10. Harris PA, Taylor R, Minor BL, et al. The REDCAP consortium: Building an international community of software platform partners. J Biomed Inform 2019; 95:103208. doi: 10.1016/j.jbi.2019.103208. PMID: 31078660.

11. Arnold F, Burns M, Mahmood K, et al. Endemic human coronaviruses in hospitalized adults with communityacquired pneumonia: Results from the Louisville Pneumonia Study. Univ Louisville J Respir Infect 2020; 4(1):Article 1. doi: 10.18297/rgh/vol4/iss1/1.
12. Price-Haywood EG, Burton J, Fort D, Seoane L. Hospitalization and mortality among black patients and white patients with COVID-19. N Engl J Med 2020; 382(26):2534-43. doi: 10.1056/NEJMsa2011686. PMID: 32459916.

13. Garg S, Kim L, Whitaker M, et al. Hospitalization rates and characteristics of patients hospitalized with laboratoryconfirmed coronavirus disease 2019 - COVID-NET, 14 states, March 1-30, 2020. MMWR Morb Mortal Wkly Rep 2020; 69(15):458-64. doi: 10.15585/mmwr.mm6915e3. PMID: 32298251.

14. Bohn B. The incidence of common respiratory viruses during the covid-19 pandemic: Results from the Louisville COVID-19 Epidemiology Study. Univ Louisville J Respir Infect 2020; 4(1):Article 58. doi: 10.18297/jri/vol4/iss1/58.

15. Docherty $A B$, Harrison $E M$, Green $C A$, et al. Features of 20133 UK patients in hospital with COVID-19 using the ISARIC WHO clinical characterisation protocol: Prospective observational cohort study. BMJ 2020; 369:m1985. doi: 10.1136/bmj.m1985. PMID: 32444460.

16. Bordon J, Chung D, Krishnan P, Carrico R, Ramirez JA. The importance of cycle threshold values in the evaluation of patients with persistent positive PCR for SARS-CoV-2: Case study and brief review. Univ Louisville J Respir Infect 2020; 4(1):Article 54. doi: 10.18297/jri/vol4/iss1/54.

17. Wölfel R, Corman VM, Guggemos W, et al. Virological assessment of hospitalized patients with COVID-2019. Nature 2020; 581(7809):465-9. doi: 10.1038/s41586-020-2196x. PMID: 32235945.

18. Long B, Brady WJ, Koyfman A, Gottlieb M. Cardiovascular complications in COVID-19. Am J Emerg Med 2020; 38(7):1504-7. doi: 10.1016/j.ajem.2020.04.048. PMID: 32317203.

19. Lansbury L, Lim B, Baskaran V, Lim WS. Coinfections in people with COVID-19: A systematic review and meta-analysis. J Infect 2020; 81(2):266-75. doi: 10.1016/j.jinf.2020.05.046. PMID: 32473235.

20. Moore S. A patient with Escherichia coli bacteremia and COVID-19 co-infection: A case report for the Louisville COVID-19 Epidemiology Study. Univ Louisville J Respir Infect 2020; 4(1):Article 15. doi: 10.18297/jri/vol4/iss1/15.

21. Grasselli G, Zangrillo A, Zanella $A$, et al. Baseline characteristics and outcomes of 1591 patients infected with SARS-CoV-2 admitted to ICUs of the Lombardy region, Italy. Jama 2020; 323(16):1574-81. doi: 10.1001/jama.2020.5394. PMID: 32250385. 